\title{
Conversion therapy from N3 unresectable lung adenocarcinoma to radical surgery: a case report
}

\author{
Huali $\mathrm{Hu}^{1}$, Shuai Zou ${ }^{1}$, Ruofu $\mathrm{Xu}^{1}$, Yoshinobu Ichiki ${ }^{2,3}$, Dirk De Ruysscher ${ }^{4}$, Fenglei Yu ${ }^{5}$, Aihui Liao ${ }^{1}$ \\ ${ }^{1}$ Department of Thoracic Surgery, Hunan Province Dikuang Hospital, Changsha 410007, China; ${ }^{2}$ Department of Surgery, School of Medicine, \\ University of Occupational and Environmental Health, Kitakyushu, Japan; ${ }^{3}$ Department of General Thoracic Surgery, National Hospital \\ Organization, Saitama Hospital, Wako, Japan; ${ }^{4}$ Department of Radiation Oncology (Maastro Clinic), GROW School for Oncology and \\ Developmental Biology, Mastricht University Medical Center, Maastricht, The Netherlands; ${ }^{5}$ Department of Thoracic Surgery, The Second \\ Xiangya Hospital, Central South University, Changsha 410000, China \\ Correspondence to: Aihui Liao. Department of Thoracic Surgery, Hunan Province Dikuang Hospital, Changsha 410007, China. \\ Email: yufenglei@csu.edu.cn.
}

\begin{abstract}
Patients with N3 non-small cell lung cancer (NSCLC) have unresectable tumors. Although significant progress has been made in the past decades for such tumors, the average median survival time remains at 10 months. Equally dismal long-term survival rates were reported with the average 5-yearsuvival rate at $9 \%$. Here, we report on a case of a patient with locally advanced disease that was treated with conversion therapy using targeted anti-PD-1 immunotherapy with platinum-based chemotherapy. Following this therapeutic regimen, the tumor showed a reversion to $\mathrm{pN} 0$ from $\mathrm{pN} 3$ and the patient showed a progression free survival time of at least 33 months.
\end{abstract}

Keywords: Conversion treatment; immunotherapy neoadjuvant treatment; N3 locally advanced NSCLC

Submitted Sep 18, 2019. Accepted for publication Sep 23, 2019.

doi: 10.21037/atm.2019.09.113

View this article at: http://dx.doi.org/10.21037/atm.2019.09.113

\section{Introduction}

It is well established that one-third of patients with nonsmall cell lung cancer (NSCLC) present with locally unresectable disease at the time of diagnosis. The standard of care for these patients is concurrent chemoradiotherapy (CRT) when EGFR mutations and anaplastic lymphoma kinase (ALK) rearrangements are absent. Although curative outcomes are achieved in patients given concurrent CRT, only a small proportion of patients achieve longterm survival, and $15-40 \%$ show recurrence that leads to treatment failure (1). According to the IASC data released in 2015, patients with pN3 NSCLC were reported to have an average median survival time of 10 months and an average 5-year-survival of $9 \%$ (2).

The use of antibodies against programmed cell death 1 (PD-1) is a promising new therapy for advanced cancers (3). In patients enrolled in the KEYNOTE-001 trial who had previously untreated NSCLC and a PD-L1 tumor proportion score of $50 \%$ or greater, pembrolizumab was associated with a response rate of $58.3 \%$, with a median progression-free survival (PFS) of 12.5 months (4). Here, we report on a successful conversion from pN3 NSCLC to $\mathrm{pN} 0$ in a patient treated with a PD-1 checkpoint inhibitor combined with platinum-based chemotherapy achieving at least 33 months' PFS.

\section{Case presentation}

In November 2016, a 62-year-old male with a 1,200 cigarette-pack-year history presented with a palpable right supraclavicular lymph nodule. The patient's first PET/CT showed 1 right upper lobe nodule associated with hilar, lower paratracheal, upper paratracheal, and right supraclavicular lymph nodes (Figure 1). An excisional biopsy of the right supraclavicular lymph node showed metastatic poorly differentiated adenocarcinoma lung cancer. Moreover, PDL-1 expression was 90\% (Figure 1). 

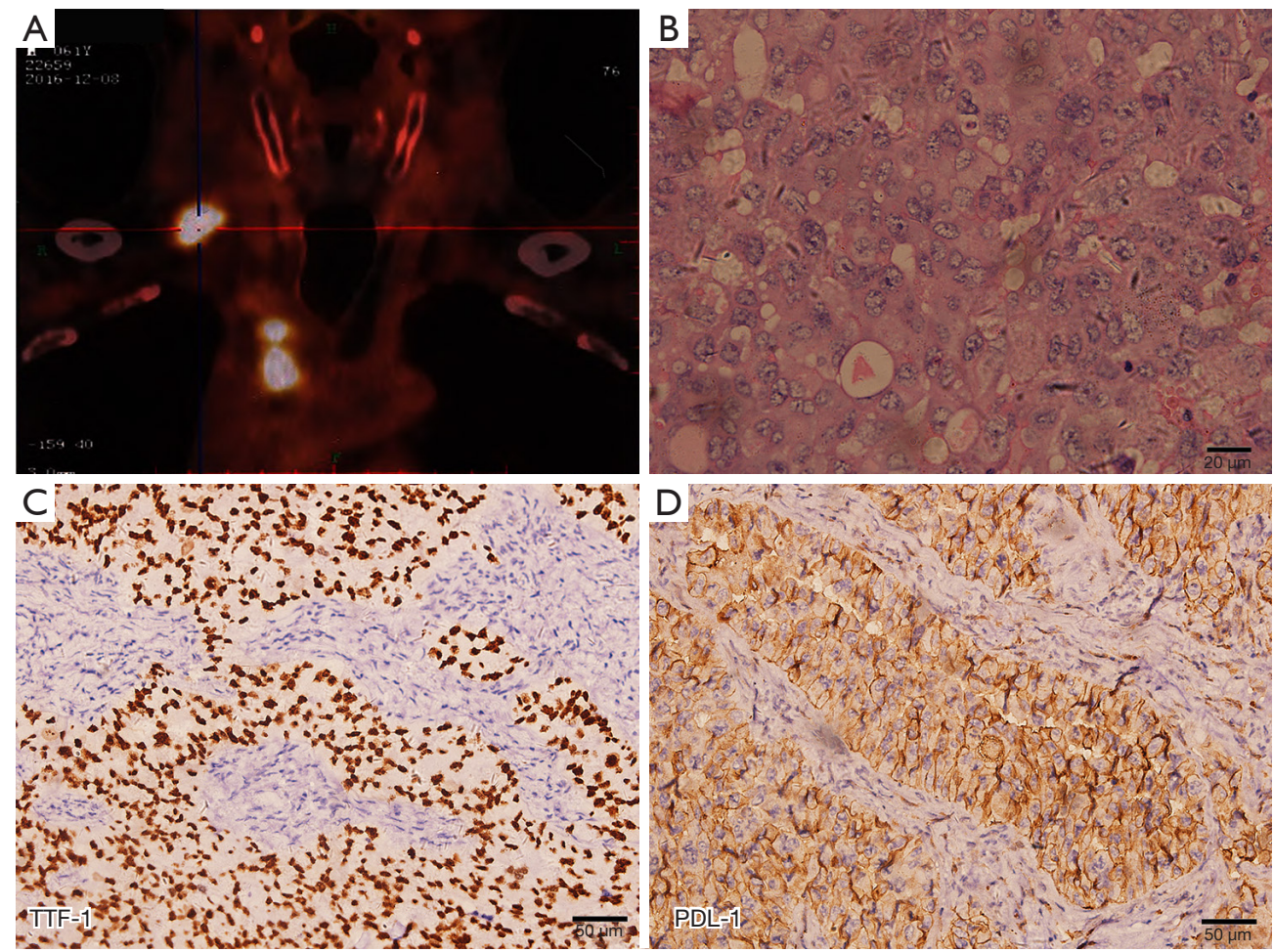

Figure 1 Pathological examination of the right supraclavicular nodule. PET/CT scan image of the right mediastinal and supraclavicular lymph nodes (A); HE-stained image of the excised supraclavicular lymph nodes (B); TTF-1 (C) and PDL-1 (D) expression of excised supraclavicular lymph nodes, respectively.

Molecular pathologic analysis results showed that EGFR mutations and ALK rearrangement were negative, while another 13 somatic mutations were detected with a high tumor mutation burden of 11.0 Muts/Mb (GeneplusBeijing, Beijing, China). He was staged T1bN3M0 (stage IIIB) according to the AJCC 8th edition cancer staging manual, with a predicted median survival of 10 months (2).

The patient was started on pemetrexed $500 \mathrm{mg} / \mathrm{m}^{2}$ body surface area and carboplatin (area under the curve, 5) combination with pembrolizumab $(200 \mathrm{mg})$ given every 3 weeks for 2 cycles. After the 2nd cycle, a surveillance PET/ CT showed a decrease in size and metabolic activity of both the lower paratracheal lymph nodes, the upper paratracheal lymph nodes, and the right upper lobe nodule. Also, both the hilar and the right supraclavicular lymph nodes disappeared with no lesions and metabolism boost (Figure 2). Toxic side effects were mild and endurable. Because of the excellent anti-tumor effect and mild side effect, an additional 2 cycles of treatment were given. In April 2017, a repeat PET/CT revealed relative disease stability compared with the disease status in February 2017 (Figure 2). The right upper lesion showed a slight decrease in size and metabolic activity in the upper paratracheal lymph nodes, while the lower paratracheal lymph nodes remained the same.

Considering the potential drug resistance, radical surgery was performed to remove the upper lobe, the right hilar/mediastinal lymph node, and the supraclavicular lymph node. Postoperative pathological examination showed that there were a few cancer cells in the primary lung cancer lesion with an abundance of tumorinfiltrating lymphocytes and proliferation of fibrous tissue (Figure 3); no cancer cells were found in the 39 excised lymph nodes (0/39). Immunohistochemical results of the primary lung cancer tissue showed that PDL-1 expression was negative. Molecular pathologic analysis of these tissue samples showed no gene mutation (Figure 3). The patient was discharged from hospital after recovery without any periprocedural complications. As of now, the patient is in follow-up without adjuvant treatment, and recent PET-CT examination has found no evidence of recurrence or metastasis (Figure 3). 

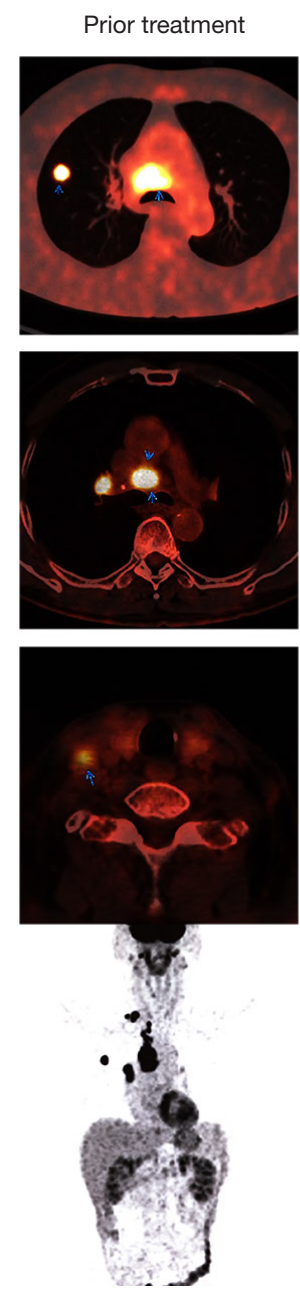
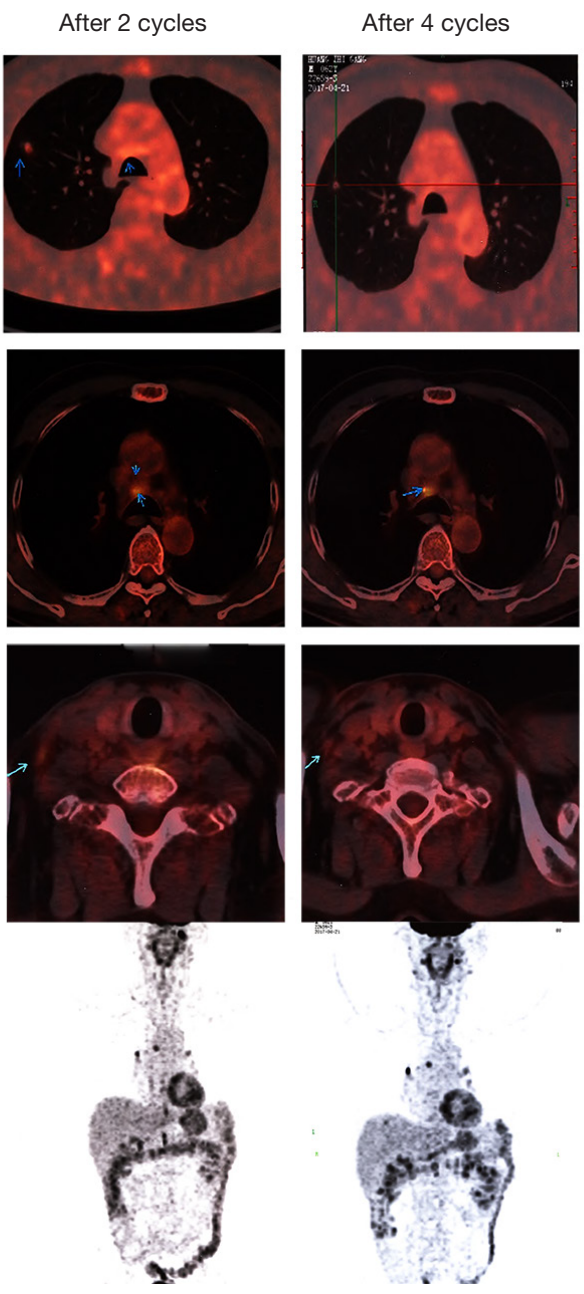
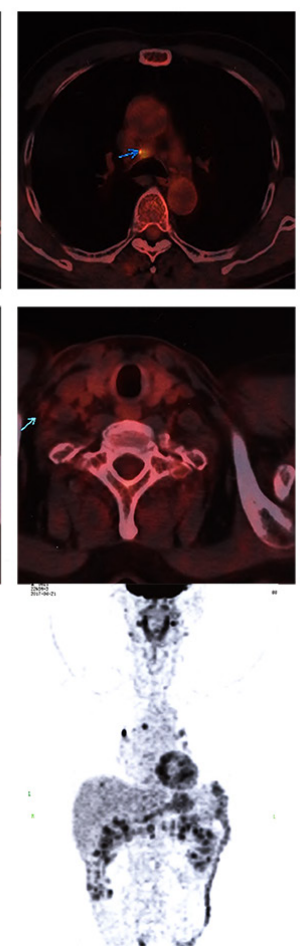

Diameter $(\mathrm{mm})$

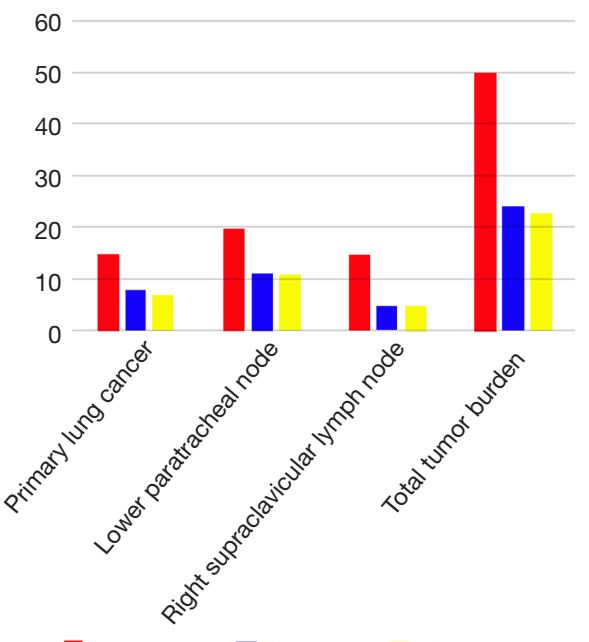

Prior treatment $\square$ After 2 cycles $\square$ After 4 cycles

Figure 2 Radiological evaluation of the treatment efficacy with PET/CT at different time points.

\section{iMDT discussion}

We conducted intradisciplinary and multidisciplinary consultation of this case. Our patient had IIIB-NSCLC, involving clustered mediastinal lymph nodes as well as supraclavicular lymph node metastasis. He had no chance of surgery according to current clinics guide, while he obtained radical surgery following chemotherapy plus immunotherapy and received sound therapeutic effect with no less than 33-month PFS. However, whether this strategy lead to a new treatment mode for IIIB-NSCLC, should also be considered.

\section{Department of Oncology}

In this case, chemotherapy plus immunotherapy successfully transformed a clinically locally advanced NSCLC patient (cT1N3M0 IIIB) to an early-stage NSCLC patient (ypT1N0M0 IA). The dramatic major pathological response (MPR) of locally advanced NSCLC neoadjuvant therapy has rarely been achieved in terms of either single-agent chemotherapy or single-agent immunotherapy. Langer et al. (5) reported that chemotherapy, in combination with pd1 blockage, could improve the objective response rate for patients with advanced non-squamous NSCLC, showing that PD-1 inhibitor could be synergistic with chemotherapy. Also, two biomarkers for PD-1 inhibitor, PDL-1 expression, and tumor mutation burden, were high. Thus, we recommended a combination of pembrolizumab, carboplatin, and pemetrexed as first-line treatment; the dramatic MPR verified the conversion from an unresectable case to a resectable case. For a long time, the radical 

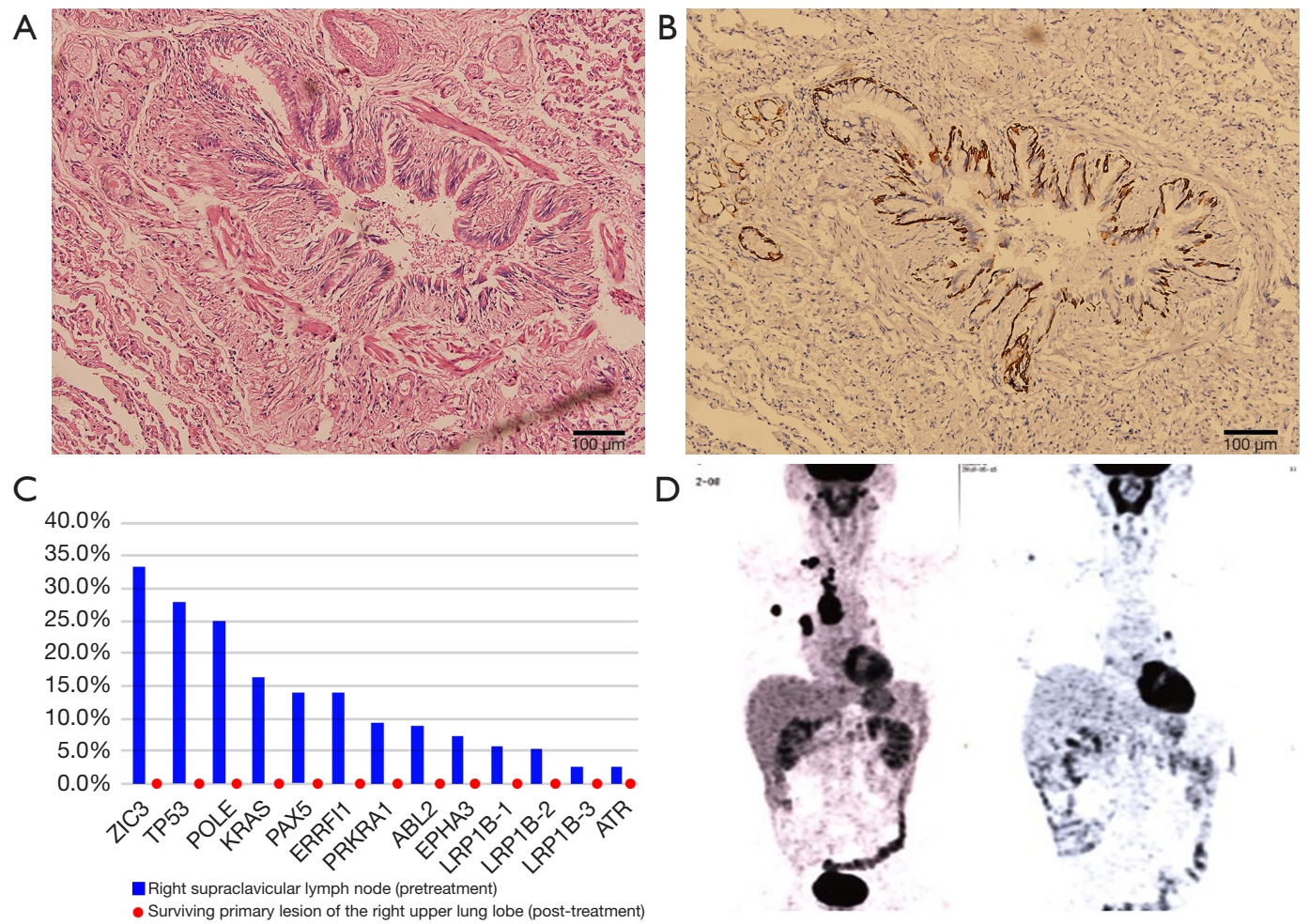

Figure 3 Postoperative pathological HE-stained picture of the surviving primary lesion of the right upper lung lobe (A). CK expression of the surviving primary lesion of the right upper lung lobe (B). The 1021-gene panel analysis results of the tissue sample from the right supraclavicular nodule (blue) and the surviving primary lesion (red), respectively (C). PET/CT scan image of the patient before treatment and 2-year postoperative follow-up (D).

operation was impossible for patients with N3 NSCLC. These patients were reported to have an average median survival time of 10 months, and an average 5-year-survival of $9 \%$ (2). The advent of immunotherapy offers a glimmer of hope for advanced lung cancer and has demonstrated an excellent down-stage effect for certain advanced cases of NSCLC. However, no experience was reported about the safety and validity of pulmonary resection in N3 NSCLC patients who have received immunotherapy. This case showed that chemotherapy plus immunotherapy could convert some patients with unresectable N3 NSCLC to early-stage NSCLC. While we should select patients by biomarkers carefully to guarantee definite downstage effect.

\section{Department of Thoracic Surgery}

This case convinced us that it is safe to perform surgery following immunotherapy plus platinum-based chemotherapy, and the medium effect appeared encouraging with a PFS of at least 33 months. This experience convinced us that a subset of unresectable advanced NSCLC patients could receive help from this strategy and achieve a radical surgery opportunity. It is worth mentioning that PDL1 expression and the tumor mutational burden of the surviving primary lung cancer tissue was negative, which means that further immunotherapy might be invalid, and its curative effect has reached its limit. In terms of radiographic evaluation, the disease presents as stable. This provides a theoretical foundation for the idea that surgical intervention should be adopted when disease stability is observed, and a partial response is noted. Postoperative adjuvant therapy was not suggested for conversion therapy from cT1N3M0 to ypT1N0M0 was obtained. We believe that this strategy could be implemented to those targeted therapy gene mutation wild-type locally advanced NSCLC patients who have both high PDL-1 expression and TMB, with the best timing of the operation being when the disease is stable following partial response, and follow-up 
observation is sufficient when MPR is achieved.

\section{There are several questions should be further discussed in this case}

Question 1: how should we select those III-NSCLC patients who can benefit from immunoadjuvant therapy and down staging enough to have the radical operation? Expert opinion 1: Dr. Yoshinobu Ichiki

We reported that the Eastern Cooperative Oncology Group performance status (ECOG PS), pathological type, standardized uptake value (SUV) on positron emission tomography (PET), white blood cell count (WBC), neutrophil, NLR (neutrophil-to-lymphocyte ratio), LDH (lactate dehydrogenase) and albumin were independently prognostic factors (6). As mentioned, PD-L1 expression, TMB and TIL might be standard biomarkers, but not complete factors.

\section{Expert opinion 2: Dr. Dirk De Ruysscher}

The treatment with chemo-immunotherapy before surgery, either in resectable or in non-resectable NSCLC is an experimental treatment. There are few prospective data available and it is unknown what the high rates of major pathological remissions and complete remissions mean for the survival of patients. It is simply unknown if a pathological remission after chemo-immunotherapy is biologically the same as after chemotherapy alone. It may well be that in order to obtain a long-lasting anti-cancer immune effect, the bulk of the tumor should remain longer in the body.

The radiological assessment of response after chemoimmunotherapy has not been settled. In all prospective studies, there has been no correlation at all between RECIST responses and pathological effects. A sizable minority of patients may even show some volume increase of the tumor without viable tumor cells in the resection specimen.

The surgical morbidity is largely unknown. Although resection seems safe, some studies report a difficult resection at the lung hilum because of fibrosis and a high rate of conversion from VATS to open thoracotomy.

Finally, there are no data to support the use of TKI in patients with stage III NSCLC as part of a curative intent treatment, even if targetable driver EGFR mutations or ALK translocations are present.

This case report is interesting, but should not lead to a different strategy than concurrent chemotherapy and radiotherapy, followed by durvalumab in fit patients with locally advanced NSCLC. Enrolment of patients like the one in this case report in prospective clinical trials is mandatory.

Question 2: how should we arrange immunoadjuvant therapy?

\section{Expert opinion 1: Dr. Yoshinobu Ichiki}

It is thought that durvalumab after CRT is a recent standard therapy for locally advanced stage III NSLC patients. In Pacific trial, PFS and OS of durvalumab after CRT for locally advanced stage III NSLC patients were 16.8 months and more than 3 years, respectively. We should weigh the data to make a therapeutic strategy for locally advanced stage III NSLC patients.

\section{Expert opinion 2: Dr. Dirk De Ruysscher}

The only proven benefit for overall survival is concurrent chemotherapy and radiotherapy, followed by durvalumab in fit patients with locally advanced NSCLC. It is important to emphasize the significant role of support care in this setting. Durvalumab should be initiated within 6 weeks after the end of radiotherapy.

\section{Question 3: how do we determine the optimal surgical} time?

\section{Expert opinion 1: Dr. Yoshinobu Ichiki}

If we apply salvage surgeries to locally advanced stage III NSLC patients, we had better confirm resistance for previous therapy without distant metastasis. We should decide whether indication of curative surgery exist or not at the first evaluation. As you know, salvage surgery is a just optional therapy to aim curative status intensively based on the patient's request.

\section{Expert opinion 2: Dr. Dirk De Ruysscher}

As mentioned previously, surgery after or before immune therapy is at present an experimental therapy, for no longterm survival data are available. Therefore, the only way to answer this question correctly is to enter patients in welldesigned prospective studies.

Question 4: is post-operative adjuvant therapy effective when MPR is achieved?

\section{Expert opinion 1: Dr. Yoshinobu Ichiki}

We think that the tumor status of just before surgery is important. If the tumor cells have resistance for previous therapy, it is difficult to expect the effect of it. We can evaluate preoperative status of the tumor cells from preoperative clinical course of image findings and pathological findings of surgical specimens. If we confirm MPR in surgical specimens, 
we should add adjuvant therapy using the same agent.

\section{Expert opinion 2: Dr. Dirk De Ruysscher}

At present, it could be argued that postoperative radiotherapy could be administered in case of persistent $\mathrm{N} 2$ disease and/or an incomplete resection.

\section{Conclusions}

Patients with drive-gene-negative N3-NSCLC were considered inoperable in most cases. Furthermore, only a very small portion of patients who were assessed as being downstage while also being able to undergo a complete excision following neoadjuvant treatment by an MDT could obtain the possibility to undergo radical surgery. Immunotherapy in combination with chemotherapy could enhance the major pathology response rate in patients with but not limited to these following factors: PD-L1 high expression, high tumor mutation burden and wild-type drive gene. For these drive-gene-negative N3-NSCLC patients, this treatment strategy could improve the downstage rate and eventually more patients could obtain radical surgery opportunity.

\section{Acknowledgments}

None.

\section{Footnote}

Conflicts of Interest: The authors have no conflicts of interest to declare.

Ethical Statement: The authors are accountable for all aspects of the work in ensuring that questions related to the accuracy or integrity of any part of the work are appropriately investigated and resolved. Written informed consent was obtained from the patient for publication of this Case Report and any accompanying images.

\section{References}

1. Furuse K, Fukuoka M, Kawahara M, et al. Phase III study of concurrent versus sequential thoracic radiotherapy in combination with mitomycin, vindesine, and cisplatin in unresectable stage III non-small-cell lung cancer. J Clin Oncol 1999;17:2692-9.

2. Asamura H1, Chansky K, Crowley J, et al. The International Association for the Study of Lung Cancer Lung Cancer Staging Project: Proposals for the Revision of the N Descriptors in the Forthcoming 8th Edition of the TNM Classification for Lung Cancer. J Thorac Oncol 2015;10:1675-84.

3. Topalian SL, Hodi FS, Brahmer JR, et al. Safety, activity, and immune correlates of anti-PD-1 antibody in cancer. $\mathrm{N}$ Engl J Med 2012;366:2443-54.

4. Hui R, Gandhi L, Carcereny E, et al. Long-term OS for patients with advanced NSCLC enrolled in the KEYNOTE-001 study of pembrolizumab (pembro) J Clin Oncol 2016;34:9026. abstract.

5. Langer CJ, Gadgeel SM, Borghaei H, et al. Carboplatin and pemetrexed with or without pembrolizumab for advanced, non-squamous non-small-cell lung cancer: a randomised, phase 2 cohort of the open-label KEYNOTE-021 study. Lancet Oncol 2016;17:1497-508.

6. Ichiki Y, Taira A, Chikaishi Y, abstract. Prognostic factors of advanced or postoperative recurrent non-small cell lung cancer targeted with immune check point inhibitors. J Thorac Dis 2019;11:1117-23.
Cite this article as: $\mathrm{Hu} \mathrm{H}, \mathrm{Zou} \mathrm{S}, \mathrm{Xu}$ R, Ichiki $\mathrm{Y}$, De Ruysscher D, Yu F, Liao A. Conversion therapy from N3 unresectable lung adenocarcinoma to radical surgery: a case report. Ann Transl Med 2019;7(20):590. doi: 10.21037/ atm.2019.09.113 